\title{
Capture-recapture population growth rate as a robust tool against detection heterogeneity for population management
}

\author{
Lucile Marescot, ${ }^{1,5}$ Roger Pradel, ${ }^{1}$ Christophe Duchamp,${ }^{2}$ Sarah Cubaynes, ${ }^{1}$ Eric Marboutin, ${ }^{3}$ \\ Rémi Choquet, ${ }^{1}$ Christian Miquel, ${ }^{4}$ and Olivier Gimenez ${ }^{1}$ \\ ${ }^{1}$ Centre d'Ecologie Fonctionnelle et Evolutive, UMR 5175, 1919 Route de Mende, F-34293 Montpellier, France \\ ${ }^{2}$ Office National de la Chasse et de la Faune Sauvage, CNERA Prédateurs et Animaux Déprédateurs, \\ Parc Micropolis, F-05000 Gap, France \\ ${ }^{3}$ Office National de la Chasse et de la Faune Sauvage, ZI Mayencin, 5 Allée de Béthléem, F-38610 Gières, France \\ ${ }^{4}$ Laboratoire d'Ecologie Alpine, UMR CNRS 5553, Université J. Fourier, BP 53, F-38041 Grenoble, France
}

Abstract. Managing large carnivores is one of the most controversial issues in wildlife conservation, as the sociopolitical challenges it raises are as important as the biological ones. Such controversial issues in wildlife conservation require objective biological components to be implemented within the management decision process, in particular, a reliable way of estimating trends in abundance. However, these species usually exhibit territoriality, low densities, and social constraints that can generate individual detection heterogeneity (IDH) of methodological (sampling) or biological (social status, marking behavior) origin. If not accounted for, IDH can lead, in turn, to strong bias in the estimation of population abundance. As a complement to population size, we propose to use the population growth rate $(\lambda)$ estimated with capture-recapture (CR) data, a robust method to detect and account for IDH, to monitor and manage elusive species. As a case study, we consider the natural recovery of the wolf (Canis lupus) population in France, for which a previous study has shown strong IDH leading to a $27 \%$ underestimation of abundance. Analyzing a nine-year data set based on a noninvasive sampling protocol relying on wolf scat genotyping, we adopt a new CR approach to estimate $\lambda$ while explicitly accounting for IDH. The annual population growth rate was estimated at $1.271 \pm 0.087$ (mean \pm SE) vs. $1.270 \pm 0.095$ when not accounting for IDH, indicating that $\lambda$ is much less sensitive to IDH than is abundance. We evaluated the robustness of our approach in a simulation study using increasing levels of IDH. The bias in $\lambda$ increased with detection heterogeneity but was low whether we used a model with IDH or not. Finally, we discuss the management implications of our findings in terms of sampling protocols and management strategies of elusive species.

Key words: Canis lupus; capture-recapture; DNA microsatellites; France; genetic testing; individual detection heterogeneity, IDH; mixture models; noninvasive methods; population growth rate; robustness; wolf.

\section{INTRODUCTION}

Most of the large, wide-ranging carnivores face high extinction risks because of anthropogenic disturbances causing the deterioration of wild habitats, isolating small populations and making them sensitive to environmental and demographic stochasticity (Rabinowitz and Zeller 2010). However, large-carnivore conservation is challenging because of its interactions with human activities. In particular, wolf (Canis lupus) management is one of the most controversial issues in wildlife conservation because public opinions are highly polarized (Treves et al. 2004). In Western Europe, this species approached extinction at the end of the 19th century (Breitenmoser 1998), surviving in fragmented relictual populations in central Italy until the 1970s (Zimen and

Manuscript received 4 December 2010; revised 6 April 2011; accepted 28 April 2011. Corresponding Editor: T. R. Simons.

${ }^{5}$ E-mail: lucile.marescot@cefe.cnrs.fr
Boitani 1975) and Spain (Blanco et al. 1992). As a consequence of international laws for wolf protection, the species started recovering both southward and northward, and reached the Alpine range in the early 1990s. Noninvasive molecular tracking (Fabbri et al. 2007) and direct observation of GPS-equipped animals (Ciucci et al. 2009) showed evidence of a natural recolonization through the Apennine, a mountain chain extending $1200 \mathrm{~km}$ along peninsular Italy.

The French government complied with the Bern convention in 1979 and in 1992 signed the European Habitat Directive (European Commission Environment 1992), which classified the wolf species as strictly protected. The recovery of the wolf population in the French Alps has created strong interactions with agricultural activities in a landscape traditionally devoted to intensive sheep breeding ( $>900000$ heads in summer). Since 1992, when the first wolf occurrences in France were confirmed, the French government started monitoring the wolf presence for conservation 
purposes while dealing with damages to livestock. Since 1995, prevention methods against livestock depredations, such as confining sheep in the presence of guarding dogs, have been proposed. As a response to social pressure and to increasing numbers of depredations, a lethal control started in 2001 while ensuring that the population viability was not threatened (Chapron et al. 2003). In this context, careful monitoring of the wolf population is crucial to assess the species conservation status resulting from management decisions and requires protocols for collecting data, as well as robust indicators of population trends.

The monitoring of elusive and wide-ranging populations of large carnivores is often based on noninvasive sampling methods such as camera trapping (Karanth et al. 2006) or noninvasive molecular tracking (Taberlet and Luikart 1999), because neither requires physical captures. The French National Game and Wildlife Agency (ONCFS) runs a noninvasive approach based on molecular tracking from scat sampling (Valière et al. 2003) to monitor the population of wolves. These genetic tags are used to estimate population size using capture-recapture (CR) methods (for a review, see Lukacs and Burnham 2005), which is in turn employed as a measure of population status (Mondol et al. 2009). However, standard CR models assume homogeneous detection probabilities among individuals, which is commonly violated in many demographic studies of various species (Ebert et al. 2010). For instance, individual heterogeneity in the detection process has been reported in several species, and is related to behavioral traits that may change with environmental and historical conditions, such as individual experience (Coleman 1998). Although incorporating individual covariates such as personality (Coleman 1998), sex (Tavecchia et al. 2001), age (Crespin et al. 2006), or social status (Summerlin and Wolfe 1973) is possible in CR models, most of these indicators of individual variability cannot be measured using noninvasive genetic protocols (Skalski et al. 1993). However, population size can be strongly biased when individual detection heterogeneity (IDH) is ignored (e.g., Harmsen et al. 2010). The higher the IDH, the stronger the bias is (Carothers 1973).

A previous study of the French wolf population showed a $27 \%$ underestimation of population size when IDH was ignored (Cubaynes et al. 2010). Prior to making decisions, managers need to account for such bias in the estimation of abundance or find an alternative demographic indicator.

We argue that the population growth rate $(\lambda)$ is a relevant demographic indicator to assess the effects of management rules and decisions on the conservation status of the population and could be used as a complement to population size. Several approaches are available to calculate $\lambda$. First, the comparison of two successive annual abundance estimates from census data is a commonly used empirical method to calculate $\lambda$. An alternative analytical method involves estimation of an asymptotic growth rate by extracting the dominant eigenvalue of a Leslie projection matrix (Caswell 2001). Realized and asymptotic $\lambda$ are equivalent only if the population is at a stationary state. Based on CR data, an alternative approach was developed to calculate $\lambda$, which consists of summarizing the population inflows and outflows through the estimation of apparent survival rates (death and emigration) and recruitment rates (birth and immigration) (Pradel 1996). This approach gives a direct estimate of the rate of increase of a population, based on time-specific estimates of survival and recruitment, along with estimates of the vital rates with the strongest effect on $\lambda$. The CR approach to estimate realized population growth rate has been widely used in birds (Franklin et al. 2004), mammals (Clark et al. 2005), reptiles (Lowe et al. 2008), fishes, and mollusks (Villella et al. 2004). This method has only recently been extended to cope with IDH. Specifically, Pradel et al. (2009) developed CR mixture models (sensu Pledger et al. 2003) in which distinct classes of heterogeneity are considered.

Here, we show that the estimation of the population growth rate is robust to IDH. First, we analyzed the French wolf data to estimate $\lambda$ using CR models accounting for IDH. Second, we performed a simulation study to estimate the bias in the population growth rate estimator when IDH was artificially generated. The objective was mainly to provide a general demonstration of the robustness of $\lambda$ to IDH under several scenarios with contrasting degrees of heterogeneity. Finally, we discuss the benefits of using a robust tool in terms of population monitoring and management.

\section{Material And Methods}

\section{Data collection}

The wolf monitoring is carried out by the ONCFS based on a dual sign survey framework: (1) An extensive survey is conducted during the year by a network of wolf experts who are dispatched to cover the alpine range and report signs of presence. (2) An intensive sign survey is stratified within all previously detected wolf territories using standard snow-tracking and wolf-howling techniques (Marboutin and Duchamp 2005), which allows the collection of scats, hairs, tissues, or urine used in DNA analyses. Individual genotypes were identified using a set of seven DNA microsatellites and a multitube approach. For every sample, each microsatellite locus was amplified eight times (Taberlet and Luikart 1999). To assess genotype reliability and minimize errors, we used a quality index corresponding to the mean frequency of the consensus genotype among the eight replicates at each locus (Miquel et al. 2006). We discarded genotypes with average quality index $<0.4$.

Among the 1167 samples collected, 840 were successfully genotyped, from which 160 individuals were identified (Table 1). We partitioned the data set into 35 three-month intervals, from spring 1995 to autumn 
TABle 1. Structure of the wolf genetic data set, showing annual distribution of samples, number of genotypes detected, and the percentage of individuals detected only once.

\begin{tabular}{cccc}
\hline \hline Year & $\begin{array}{c}\text { No. } \\
\text { samples }\end{array}$ & $\begin{array}{c}\text { No. genotypes } \\
\text { detected }\end{array}$ & $\begin{array}{c}\text { Detected only } \\
\text { once in a year (\%) }\end{array}$ \\
\hline 1995 & 13 & 4 & 25 \\
1996 & 46 & 12 & 33 \\
1997 & 90 & 21 & 52 \\
1998 & 161 & 30 & 33 \\
1999 & 116 & 31 & 52 \\
2000 & 110 & 25 & 44 \\
2001 & 113 & 38 & 60 \\
2002 & 286 & 69 & 52 \\
2003 & 240 & 66 & 54 \\
\hline
\end{tabular}

Note: The number of samples (indices) includes the number of scats, hairs, urine, and blood collections used in the DNA analyses.

2003. Up to $60 \%$ of individuals were detected only once, whereas some detected more than 45 times.

\section{Estimating survival and recruitment with IDH}

We performed two distinct analyses. First, we estimated the survival probability $\varphi_{t}$, the probability that an individual alive in year $t$ survived to the next year and remained in the study area. Second, we estimated the seniority probability $\gamma_{t}$, the probability for an individual present at a given occasion $t$ to be already present at the previous occasion, which is also defined as $1-f_{t}$, where $f_{t}$ stands for the recruitment rate. We assumed that there was no misidentification.

In both analyses, we incorporated IDH in the model by assuming that individuals were distributed in two detectability classes (high and low recapture probability). Such models belong to the family of mixture models (Pledger et al. 2003), which were reformulated using a hidden structure corresponding to individuals that belong to a specific IDH state (Pradel et al. 2009). Depending on the frequency of detections, an individual can be assigned to three underlying states: alive with a high detection probability ("H"), alive with a low detection probability ("L"), or dead ("D"). Two observations were possible: "1" for a detection and " 0 " for otherwise.

For example, the encounter history "1101" corresponds to an individual present and encountered in the first, second, and last sampling occasions, but missed in the third one. When accounting for IDH with timedependent parameters, its probability is

$$
\begin{aligned}
P(1101)= & \pi \varphi_{1} p_{2}^{\mathrm{L}} \varphi_{2}\left(1-p_{3}^{\mathrm{L}}\right) \varphi_{3} p_{4}^{\mathrm{L}} \\
& +(1-\pi) \varphi_{1} p_{2}^{\mathrm{H}} \varphi_{2}\left(1-p_{3}^{\mathrm{H}}\right) \varphi_{3} p_{4}^{\mathrm{H}}
\end{aligned}
$$

where $\pi$ is the probability that an individual belongs to state $\mathrm{L}$ and $p^{\mathrm{L}}$ and $p^{\mathrm{H}}$ are the detection probabilities in the $\mathrm{L}$ and $\mathrm{H}$ detection classes.

In a second step, we estimated seniority by reversing the encounter histories (Pradel 1996). If we consider again the same individual, it has a reversed history 1011. Given that it was last detected at occasion 4 , it was already present at occasion 3 with probability $\gamma_{4}$, was detected at occasion 3 , and was already present at time 2 with probability $\gamma_{3}$, was missed at occasion 2 but was already alive and detected at occasion 1 , which gives

$$
\begin{aligned}
P(1101)= & v \gamma_{4} p_{3}^{\mathrm{L}} \gamma_{3}\left(1-p_{2}^{\mathrm{L}}\right) \gamma_{2} p_{1}^{\mathrm{L}} \\
& +(1-v) \gamma_{4} p_{3}^{\mathrm{H}} \gamma_{3}\left(1-p_{2}^{\mathrm{H}}\right) \gamma_{2} p_{1}^{\mathrm{H}}
\end{aligned}
$$

where $v$ is the proportion of individuals in the detection class $\mathrm{L}$.

\section{Joint analysis of population growth rate}

To estimate $\lambda$, we considered a joint model parameterized in terms of recruitment, survival, and detection probabilities. The number of individuals at time $t$ that have survived can be expressed as $N_{t} \varphi_{t}$, which is equivalent to the number of individuals at time $t+1$ that were not recruited between $t$ and $t+1$, i.e., $N_{t+1} \gamma_{t+1}$. Setting these two quantities equal, the realized $\lambda$ can be expressed as the ratio of survival and seniority rates:

$$
\lambda_{t}=N_{t+1} / N_{t}=\varphi_{t} / \gamma_{t+1} .
$$

This formula does not explicitly involve the detection probability, which makes it valid whether or not IDH is included in the CR model.

The separate recruitment and survival analyses were carried out using program E-SURGE (Choquet et al. 2009), whereas the joint $\lambda$ analysis was implemented in MATLAB by adapting existing codes (Pradel et al. 2009).

\section{Temporal and IDH effects on survival and recruitment}

For the seniority analysis, we considered models with IDH and temporal effects on all parameters. We fitted models from the simplest CR model with constant seniority probability and constant homogeneous detection probability to the full model with time effects on the seniority probability and an interaction between time and IDH on the detection probability. Besides samplingperiod effects (from 1 April 1995 to 31 December 2003 with a three-month time step), we also tested for season (four groups with detection events gathered every four seasons in successive years) and year effects (four successive occasions gathered nine times) on the detection probability that might reflect a different sampling effort across years and seasons, with better conditions to detect signs during winter. Similar temporal effects were also tested on seniority. We also investigated models with different recruitment probabilities in both detection classes, assuming that IDH could arise from the dominance status of the wolf alpha pairs vs. subordinates.

For the survival analysis, we refer to Cubaynes et al. (2010), who analyzed the same data to estimate abundance. Models including or ignoring IDH were also considered. The same effects as previously described were investigated. 
Model selection was based on the Akaike Information Criterion corrected for small sample size $\left(\mathrm{AIC}_{\mathrm{c}}\right.$; Burnham and Anderson 2002). We additionally calculated $\mathrm{AIC}_{\mathrm{c}}$ weights to quantify the likelihood that the data are better represented by a particular model than other candidate models.

We proceeded with the joint analysis of $\lambda$ by building models ranging from the simplest (with constant survival, seniority, and detection probabilities) to the full model (with time dependence in survival and seniority rates and interaction effects of time and heterogeneity on the detection probability). All models were compared to their counterpart, ignoring IDH.

\section{Simulations}

$\lambda$ robustness to $I D H$.- - We investigated the robustness of $\lambda$ using simulations under scenarios with increasing IDH. We calculated the bias and precision in the $\lambda$ estimator when using a CR model accounting for IDH vs. a CR model ignoring IDH. If the two estimates were close, we concluded that $\lambda$ was robust to IDH. We simulated data with survival $\varphi=0.85$ and $\lambda=1$ (stable population), 1.05 (increasing population), or 0.95 (decreasing population). We generated a gradient of heterogeneity in the detection probability. At the first occasion, two cohorts of $N=50$ individuals were assigned to both detection classes. We ran the study on 10 sampling periods. At each new occasion, we added to the two detection groups as many individuals as there were individuals expected to die, i.e., $N(1-\varphi)$ (Hines and Nichols 2002) in the stable population, $N_{t-1}(1-\varphi)$ $+0.05 N_{t-1}$ in the increasing population and $N_{t-1}(1-\varphi)$ $-0.05 N_{t-1}$ in the decreasing population. These new recruits survived and were detected with the same probabilities as in the first two cohorts. Detection probability in the reference detection class was $p_{1}=$ 0.9 , and $p_{2}$ in the other class varied from 0.1 to 0.9 . By doing so, we investigated five levels of IDH from the most heterogeneous scenario $\left(p_{1}=0.9\right.$ and $\left.p_{2}=0.1\right)$ to the most homogeneous one $\left(p_{1}=p_{2}=0.9\right)$. For each scenario, we simulated 500 data sets to which we fitted two models (joint likelihood) to estimate $\lambda$, one with homogeneous detection probability $(\operatorname{model}(\varphi, p, \gamma))$ and the other with heterogeneous detection probability (model $\left.\left(\varphi, p_{\text {het }}, \gamma\right)\right)$. We looked at the bias in $\hat{\lambda}$ the estimator of $\lambda$ calculated as

$$
B(\hat{\lambda})=\frac{E(\hat{\lambda})-\lambda}{\lambda} \approx \frac{\sum_{i=1}^{500} \frac{\hat{\lambda}_{i}}{500}-\lambda}{\lambda} .
$$

To assess the precision, we calculated the mean square error $\operatorname{MSE}(\hat{\lambda})$, which can be approximated as

$$
\operatorname{MSE}(\hat{\lambda}) \approx \sum_{i=1}^{500} \frac{\left(\hat{\lambda}_{i}-\lambda\right)^{2}}{500}
$$

A low MSE is characteristic of a good trade-off between low bias and low variance.
TABLE 2. Model selection in the seniority analysis of the French wolf population (1995-2003), sorted by increasing

\begin{tabular}{|c|c|c|c|c|}
\hline \multicolumn{2}{|c|}{ Model } & \multirow[b]{2}{*}{$\mathrm{np}$} & \multirow[b]{2}{*}{$\mathrm{AIC}_{\mathrm{c}}$} & \multirow[b]{2}{*}{$\mathrm{AIC}_{\mathrm{c}}$ weight } \\
\hline Seniority & Detection & & & \\
\hline het & het $+t$ & 38 & 1341.56 & 0.752 \\
\hline het & het + season & 8 & 1344.47 & 0.175 \\
\hline hom & het $+t$ & 37 & 1347.69 & 0.035 \\
\hline hom & het + season & 7 & 1347.69 & 0.035 \\
\hline hom & het $\times$ season & 10 & 1352.83 & 0.003 \\
\hline het & het + year & 13 & 1361.65 & 0 \\
\hline hom & het + year & 12 & 1366.28 & 0 \\
\hline het & het $\times$ year & 21 & 1366.45 & 0 \\
\hline het & het & 5 & 1367.43 & 0 \\
\hline hom & het & 4 & 1370.48 & 0 \\
\hline hom & het $\times$ year & 20 & 1371.7 & 0 \\
\hline het & het $\times t$ & 70 & 1396.36 & 0 \\
\hline het & Season & 7 & 1423.23 & 0 \\
\hline het & het $\times$ season & 11 & 1430.31 & 0 \\
\hline het & $t$ & 37 & 1434.82 & 0 \\
\hline het & hom & 4 & 1443.52 & 0 \\
\hline het & year & 12 & 1446.58 & 0 \\
\hline hom & season & 5 & 1462.75 & 0 \\
\hline hom & $t$ & 35 & 1479.46 & 0 \\
\hline hom & hom & 2 & 1484.41 & 0 \\
\hline hom & year & 10 & 1492.04 & 0 \\
\hline
\end{tabular}
$\mathrm{AIC}_{\mathrm{c}}$.

Notes: The number of estimable parameters is given by "np." Akaike weights allow one to interpret the observed $\mathrm{AIC}_{\mathrm{c}}$ differences in terms of conditional probabilities for each model and to evaluate the extent to which the data are supported by a model over all other candidate models. Models incorporated individual heterogeneity (het) or homogeneity (hom) in detection and seniority probabilities, and the main effects of time ( $t$, in 3-month intervals), season, or year tested with individual heterogeneity on an additive $(+)$ or interaction $(\times)$ scale. Models with temporal effects on seniority (trimester, years, seasons) were not displayed because their AIC was uniformly higher than that for models without temporal effects on seniority. The table shows the same effects on parameters as in Cubaynes et al. (2010).

\section{Adapted simulation to mimic the wolf case study}

We carried out simulations mimicking the French wolf population case study. Although individual heterogeneity in survival was found in a previous analysis of the data (Cubaynes et al. 2010), it could not be incorporated in the joint analysis of $\lambda$. To investigate the potential bias, we simulated data with two groups of individuals with different survival probabilities using the same procedure as before. Parameter values were chosen to match the estimates that were obtained from the best model in the survival analysis (Cubaynes et al. 2010): $\varphi_{1}$ $=0.75, p_{1}=0.1, \varphi_{2}=0.9, p_{2}=0.7$ and $\pi=0.75$. We then looked at a scenario similar to the joint analysis of $\lambda$. We generated 500 data sets with 35 capture occasions using parameters matching the estimates that we obtained from the best model fitted to the wolf data set.

\section{RESUlts \\ Wolf data analysis}

Analyses of survival and recruitment.-IDH was selected by the $\mathrm{AIC}_{\mathrm{c}}$ in the seniority (Table 2) and the survival analysis (Cubaynes et al. 2010; see Appendix B). 
TABLE 3. Parameter estimates of wolf survival $(\varphi)$, seniority $(\gamma)$, population growth rate $(\lambda)$, and detection rate $(p)$ in separate and joint analyses of the best-fitting model, assuming a two-class heterogeneous (het) mixture of individuals.

\begin{tabular}{clcc}
\hline \hline Analysis type & \multicolumn{1}{c}{ Parameter } & Low detectability & High detectability \\
\hline $\begin{array}{c}\text { Survival analysis } \\
\left(\varphi_{\text {het, }} p_{\text {het }+ \text { semester }}\right)\end{array}$ & Survival, $\varphi$ & $0.753(0.54-0.94) \dagger$ & $0.901(0.717-0.984) \dagger$ \\
& Dection, $p$ & $0.22(0.11-0.37) \dagger$ & $0.86(0.74-0.96) \dagger$ \\
& Jan-Jun & $0.10(0.05-0.18) \dagger$ & $0.64(0.51-0.82) \dagger$ \\
Jul-Dec & $0.578(0.553-0.602)$ & $0.810(0.793-0.823)$ \\
$\left(\gamma_{\text {het, }} p_{\text {het }+t}\right)$ & Seniority, $\gamma$ & Range $0.006(0.001-0.046)$ & Range $0.082(0.011-0.416)$ \\
& Detection $p$, by & to $0.248(0.093-0.513)$ & $0.836(0.630-0.939)$ \\
Joint analysis & sampling occasion $t$ & & $0.839(0.765-0.913)$ \\
$\left(\varphi, p_{\text {het+season, } \gamma)}\right.$ & Survival, $\varphi$ & & $0.660(0.597-0.725)$ \\
& Seniority, $\gamma$ & $1.271(1.101-1.44)$ \\
& Growth rate $\lambda$, & & $0.576(0.487-0.665)$ \\
& Detection, $p$ & $0.072(0.011-0.133)$ & $0.369(0.294-0.445)$ \\
& Spring & $0.046(0.007-0.085)$ & $0.373(0.300-0.446)$ \\
& Summer & $0.046(0.006-0.086)$ & $0.677(0.592-0.761)$ \\
\hline
\end{tabular}

Notes: Individuals of low and high detectability classes are included in the heterogeneous mix. Semester detection probabilities are given in the survival analysis. In the seniority analysis the best model detected an effect of sampling period dependence (trimester) on the two detection probabilities. The range of detection probabilities in the two detection class is given. Values in parentheses are $95 \%$ confidence intervals.

$\dagger$ Results are from Cubaynes et al. (2010).

However, there were discrepancies in the temporal effects in the survival vs. the seniority analysis. In the latter, a model with a sampling-period effect on the detection probability was selected (recall that the time unit is a three-month period here; Table 2) while in the former, Cubaynes et al. (2010) found a semester effect.

The best model in the seniority analysis was four times as plausible as a model with season effects on the detection rate. It showed an annual seniority rate of 0.809 (95\% confidence interval: [0.793-0.823]) for the $\mathrm{H}$ class (annual recruitment rate of 0.191 ) and 0.578 ([0.553-0.602]) for individuals belonging to the L class (Table 3). Detection probabilities ranged from 0.082 ([0.011-0.416] to $0.836([0.630-0.939])$ for the $\mathrm{H}$ class and from 0.006 ([(0.001-0.046]) to 0.248 ([0.093-0.513]) in the L class (Table 3).

\section{Joint analysis of $\lambda$}

In the joint analysis, models with constant survival and seniority were consistently better ranked than those with season, trimester, and year effects. The best model accounted for an additive effect of season and IDH on the detection probability (Table 4) and was three times as plausible as the equivalent model with a season effect on the vital rates.

Annual survival $\left(\varphi=\Pi \varphi_{t}\right)$ was 0.839 ([0.765-0.913]), and the annual recruitment rate $\left(f=1-\Pi \gamma_{t}\right)$ was 0.342 ([0.264-0.420]). The detection probabilities for both $\mathrm{L}$ and $\mathrm{H}$ classes were higher in winter and spring compared to the summer and autumn season (Table $3)$. We estimated that $12.5 \%([6.8-18.2 \%])$ of the individuals were in the $\mathrm{H}$ class.

Estimates and confidence intervals of annual $\lambda$ were similar between the best model accounting for IDH
$(1.271[1.201-1.343])$ vs. the second best model excluding IDH (1.270 [1.200-1.435]).

\section{Simulations}

Five scenarios with contrasting IDH.-We found similar estimates and low bias in $\lambda$ estimated in the heterogeneous $\left(\varphi, p_{\text {het }}, \gamma\right)$ vs. homogeneous model $(\varphi, p$, $\gamma)(<0.005 \%$; Fig. 1 and Appendix A). This was verified

TABle 4. Model selection in the joint analysis of the French wolf population growth rate (1995-2003).

\begin{tabular}{lrcc}
\hline \hline \multicolumn{1}{c}{ Model } & $\mathrm{np}$ & $\mathrm{AIC}_{\mathrm{c}}$ & $\mathrm{AIC}_{\mathrm{c}}$ weights \\
\hline$\left(\varphi p_{\text {het }+ \text { season }} \gamma\right)$ & 8 & 2335 & 0.705 \\
$\left(\varphi_{\text {season }} p_{\text {het }+ \text { season }} \gamma_{\text {season }}\right)$ & 14 & 2337 & 0.259 \\
$\left(\varphi p_{\text {het }} \times\right.$ season $\left.\gamma\right)$ & 11 & 2341 & 0.035 \\
$\left(\varphi_{\text {year }} p_{\text {het }+ \text { season }} \gamma_{\text {year }}\right)$ & 22 & 2349 & 0.001 \\
$\left(\varphi_{\text {season }} p_{\text {het }} \gamma_{\text {season }}\right)$ & 11 & 2361 & 0 \\
$\left(\varphi p_{\text {het }} \gamma\right)$ & 5 & 2376 & 0 \\
$\left(\varphi_{\text {year }} p_{\text {het }} \gamma_{\text {year }}\right)$ & 19 & 2389 & 0 \\
$\left(\varphi_{t} p_{\text {het }+ \text { season }} \gamma_{t}\right)$ & 74 & 2391 & 0 \\
$\left(\varphi_{t} p_{\text {het }+t} \gamma_{t}\right)$ & 104 & 2402 & 0 \\
$\left(\varphi p_{\text {season }} \gamma\right)$ & 6 & 2467 & 0 \\
$\left(\varphi_{\text {season }} p_{\text {season }} \gamma_{\text {season }}\right)$ & 12 & 2471 & 0 \\
$\left(\varphi_{\text {season }} p \gamma_{\text {season }}\right)$ & 9 & 2484 & 0 \\
$\left(\varphi_{\text {year }} p_{\text {season }} \gamma_{\text {year }}\right)$ & 20 & 2487 & 0 \\
$\left(\varphi p_{\text {season }} \gamma\right)$ & 6 & 2467 & 0 \\
$(\varphi p \gamma)$ & 3 & 2503 & 0 \\
$\left(\varphi_{t} p_{\text {season }} \gamma_{t}\right)$ & 72 & 2513 & 0 \\
$\left(\varphi_{\text {year }} p \gamma_{\text {year }}\right)$ & 17 & 2514 & 0 \\
$\left(\varphi_{t} p_{t} \gamma_{t}\right)$ & 102 & 2526 & 0 \\
\hline
\end{tabular}

Notes: Terms are: np, number of parameters; $\varphi$, survival rate; $p$, detection probability indexed by time $t$ for sampleperiod effects (three-month interval) or by season for seasonal effects and by het for heterogeneity effects; $\gamma$, seniority rate; and $\pi$, proportion in the high-detection class. The $\mathrm{AIC}_{\mathrm{c}}$ weights allow one to interpret the observed $\mathrm{AIC}_{\mathrm{c}}$ differences in terms of conditional probabilities for each model and to evaluate the extent to which the data are supported by a model over all other candidate models. 

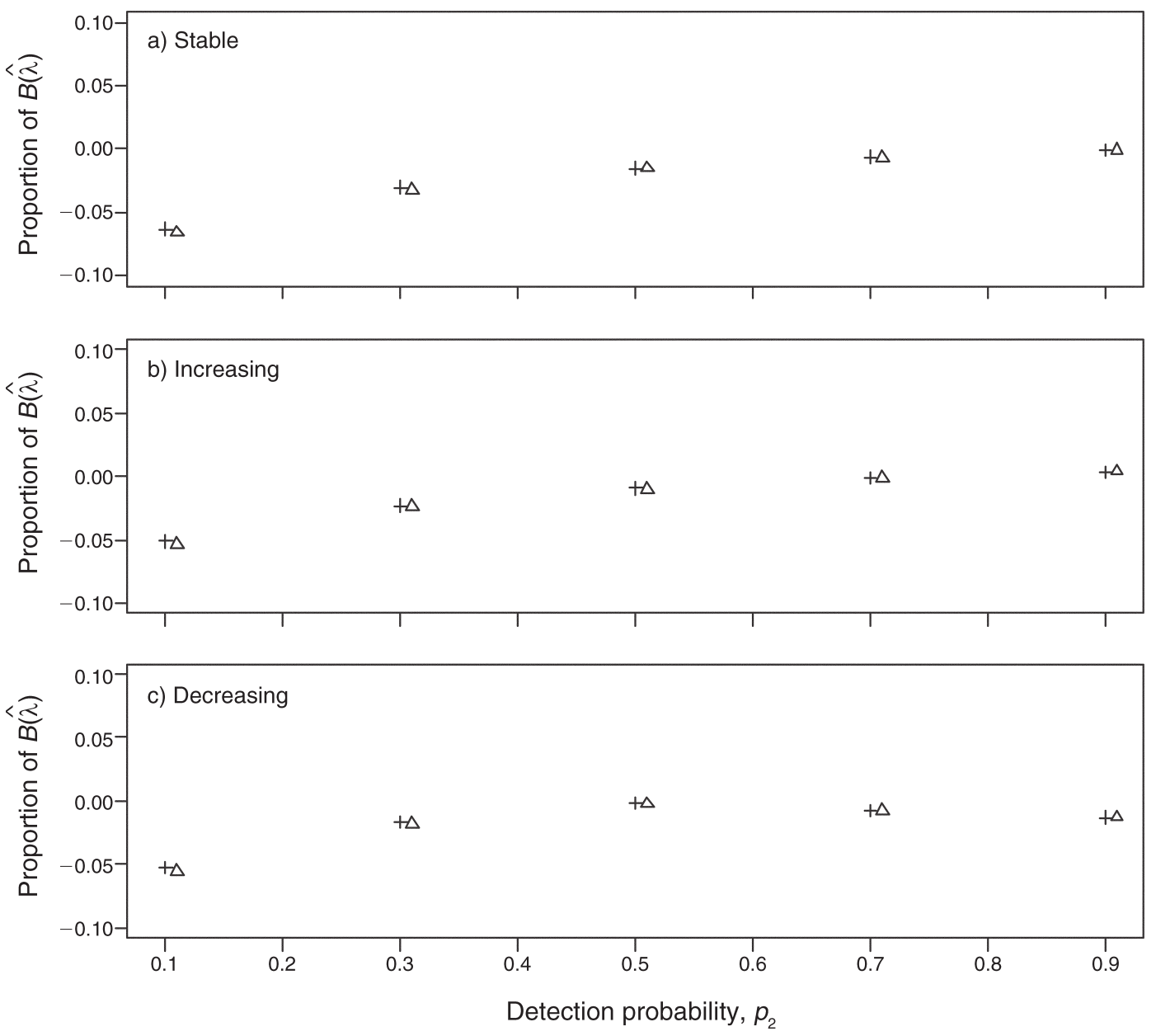

FIG. 1. Population growth rate bias $B(\hat{\lambda})$ in the heterogeneous model ( $\varphi, p_{\text {het }}, \gamma ;$ triangle symbols $)$ and the homogeneous model $(\varphi, p, \gamma$; cross symbols) for wolves in France, where $\varphi$ is survival and $\gamma$ is seniority. The homogeneous model assumed constant detection probabilities among individuals, whereas the heterogeneous model assumed that individuals were distributed in two detectability classes ( $p_{1}$ and $p_{2}$ recapture probability). In all three panels, detection probability in the reference detection class was $p_{1}=0.9$; in the other class it varied along the detection gradient, $p_{2}=(0.1,0.3,0.5,0.7,0.9)$. These models were fitted to data simulated under three scenarios: (a) $\lambda=1$ for a stable population; (b) $\lambda=1.05$ for an increasing population; and (c) $\lambda=0.95$ for a decreasing population; in all cases, $\varphi=0.85$. Mean-square errors are provided in Appendix A.

for the stable (Fig. 1a), the increasing (Fig. 1b), as well as the decreasing populations (Fig. 1c). The estimated bias increased with detection heterogeneity (Fig. 1), with the lowest value for the homogeneity scenario $(B(\hat{\lambda}) \approx$ -0.0001 for $p_{1}=p_{2}=0.9$; Appendix A), and the largest bias for the extreme heterogeneity scenario $(B(\hat{\lambda}) \approx$ -0.01 for $p_{1}=0.9, p_{2}=0.1$; Appendix A). The bias was negative except for the scenario with an increasing population and with two homogeneous groups of individuals $\left(B(\hat{\lambda}) \approx 0.0034\right.$ in $\left.\left(\varphi, p_{\text {het }}, \gamma\right) ; B(\hat{\lambda})\right) \approx$ 0.0038 in $(\varphi, p, \gamma))$ and for the scenario with a decreasing population in the cases of $p_{2}=0.7$ and $p_{2}=0.9$ (Appendix A). The patterns in the bias for survival and seniority rates were similar to what we found for $\lambda$ (Appendix B). MSE was negligible in most cases and decreased along the gradient (from the most heterogeneous to the homogeneous scenario); incorporating IDH did not increase MSE considerably (Appendix A).

\section{Simulations mimicking the case study}

Ignoring individual heterogeneity in survival caused no significant bias in growth rate $\left(B(\hat{\lambda})=-8.4 \times 10^{-3}\right.$ in the homogeneous model vs. $B(\hat{\lambda})=-6.9 \times 10^{-3}$ in the heterogeneous model). No significant bias in $\hat{\lambda}$ was found when mimicking the wolf case study. It was small and similar whether we accounted for heterogeneity or $\operatorname{not}(B(\hat{\lambda}))=1.48 \times 10^{-3}$ in the homogeneous model and $B(\hat{\lambda})=1.72 \times 10^{-3}$ and in the heterogeneous model). We found low MSE in simulations with IDH in survival $\left(\operatorname{MSE}(\hat{\lambda})=5.6 \times 10^{-6}\right.$ in the homogeneous model, $\operatorname{MSE}(\hat{\lambda})=6.8 \times 10^{-7}$ in the heterogeneous model).

\section{Discussion}

Using CR data on wolves, we provided evidence of the robustness of population growth rate to IDH, further endorsed by simulations accounting for scenar- 
ios with contrasting levels of detection heterogeneity. The simulations revealed negligible bias and low MSE in all scenarios we considered. We found a small negative bias in survival that increased as the detection heterogeneity increased, in accordance with Devineau et al. (2006). Hines and Nichols (2002) merely detected a bias in $\lambda$ when fitting a model with constant parameters and a homogeneous detection. They could not fit a model having two heterogeneous detection classes as it had not yet been developed. Our approach assesses the robustness of $\lambda$ with respect to IDH by comparing the estimators from models with homogeneous vs. heterogeneous detectability. Our results were obtained for models with constant parameters as they matched our findings for the wolf case study. Further investigations exploring alternative scenarios (e.g., time effects) would be useful to complement the robustness analysis.

From the application to the French wolf population, the joint analysis revealed that $\lambda$ was robust with respect to IDH. We found that the population annually increased by $27 \%$ whether IDH was taken into account or not. We investigated the effect of heterogeneity in survival and seniority separately because the joint analysis cannot account for it in all parameters (including detection) simultaneously (Pradel et al. 2009). However, our simulations showed that ignoring individual heterogeneity in survival and seniority was unlikely to cause bias in the estimation of $\lambda$ for the wolf case study.

IDH can result from underlying features intrinsic to the nature of the data or the species' biology and from extrinsic features of the sampling design induced by noninvasive surveys (Crespin et al. 2008). The noninvasive molecular tracking using scats always runs on borderline conditions regarding low quantity and quality of the DNA material (Taberlet et al. 1996). There is a risk of detecting false allele or allelic dropouts, which generate additional individuals detected only once (Marucco et al. 2009). A recent study on wolves (Marucco et al. 2009) using noninvasive genetic data and a multi-tube approach found that $46.3 \%$ of the initially detected genotypes had to be discarded because of allelic dropouts, false or partial null alleles. In case artifactual genotypes still remain, one can account for misidentifications explicitly in the closed-population models using recent developments in the analysis of CR data (Wright et al. 2009, Link et al. 2010). Note, however, that these methods are still to be developed for open populations to correct survival and population growth rate for identification errors.

In addition to the nature of the data, there are also biological explanations for IDH. Because animals were not captured physically, it was not possible to collect state covariates possibly underlying individual heterogeneity, e.g., social status (Summerlin and Wolfe 1973, Ebert et al. 2009, Wilson et al. 2003) or age (Davis et al. 2003, Crespin et al. 2006). Further analyses could be conducted for each scat genotyped to produce the information required and include it in the model as a covariate. However, generating relevant information at the individual level from genetic tags remains challenging. First, even though discriminating alpha pairs from their offspring in molecular tracking through kinship analysis is feasible, it would require a larger set of microsatellites allowing assignment tests (Blouin 2003). Second, information on age is difficult to extract from scat deposit and subsequent genetic analyses except where visual contact at dens or rendezvous sites during summer is possible.

Although the sampling strategy used standard protocols of snow-tracking and wolf-howling, the sampling effort outside wolf pack territories could not be quantified for logistical reasons. The monitoring was carried out across the Alps $\left(>85000 \mathrm{~km}^{2}\right)$ involving 1000 observers geographically located to optimize the spatial coverage and the chances of detecting scat. IDH is more likely to arise in such large areas because individuals that are usually difficult to detect, the dispersers, can be sampled. In contrast, Marucco et al. (2009) did not detect heterogeneity among individuals in the detection process, probably because they undertook an intense surveying effort on a small sampling coverage with few sampling occasions. More generally, in a metaanalysis of 38 studies, Ebert et al. (2009) showed that when the sampling coverage and the number of sampling occasions were low, the IDH was less likely to be detected even though it was intrinsically present.

IDH might also be related to the fact that the systematic winter tracking can overlap with favorite itineraries of some wolves having a particular marking behavior. Using trained dogs to search for feces could be a relevant strategy to overcome this issue (Smith et al. 2003), as they can find samples off trails in places difficult to reach and hence detect with higher probability those individuals from the low detectability class. Radiotelemetry could also be useful to discriminate extrinsic IDH related to scat sampling designed on line transects from intrinsic IDH related to the biology of species highly mobile, such as wolves, coyotes (Kohn et al. 1999), or bears (Boulanger et al. 2004).

Because IDH was detected in our data, it is not necessary to spend more effort in the field trying to detect IDH and its potential sources; we choose to model IDH using a CR mixture model with two hidden detection classes. However, one should remain cautious when trying to biologically interpret the detection classes; complex biological phenomena may influence detection probability. Based on common knowledge of the wolf life cycle, the highly detectable class might correspond to dominant individuals that were more likely to defecate or urinate to mark their territory in deliberately placed locations to be more detectable (Vila et al. 1994), whereas the weakly detectable individuals might include both pups and subordinates. Dispersers may also be assigned to the weakly detectable class (Cubaynes et al. 2010) because they lived mostly outside 
of the pack territory where the main survey effort occurred. In the separate analysis, $81 \%$ of the individuals alive in the $\mathrm{H}$ class at a given year were already alive the year before, suggesting that they were neither yearlings nor immigrants. Therefore the high-detection class surely represents the dominant resident adults (Mech and Boitani 2003). In the weakly detectable class, the seniority rate was $57 \%$, suggesting that this class is made up of many of the new recruits, i.e., the yearlings and the immigrants entering the population.

Seasonal effects on detection probability can be related to the species' biology and/or the study design. We found that higher detection probabilities in both the low- and high-detection classes occurred in winter. This might be explained by the presence of snow, which favors both track and scat detection. DNA quality also increases in the cold, minimizing the genetic loss before PCR runs. A possible biological explanation could be that, during summer, pups stay at home sites (den and rendezvous locations) until early fall (Harrington and Mech 1983). In winter, all of the members of the pack, including yearlings, get knit together for hunting, which would explain a higher detection probability through their scat deposit.

When wolves colonize agricultural areas that have been unoccupied for generations, socioeconomical conflicts emerge with shepherds struggling to protect their livestock from depredations, and governments devote large funds for damage prevention and compensation (Rutledge et al. 2009). Because the French wolf population is now better established than during the 1990s, and may in some places already be close to the new management concept of "social carrying capacity" (Beyer et al. 2006), some lethal control has been implemented by the French government to manage hotspots where attack rates on livestock were high, to improve social acceptance and ensure long-term population viability. These decisions often relied upon assessment of abundance and sustainable harvest rates (Patterson and Murray 2008). Since 2004, the French wolf action plan has been established to match the international requisites of population viability (Chapron et al. 2003) and to ensure a favorable conservation status $(\lambda>1$, sensu the 1992 European Habitat directive).

Overall, we see population growth rate (regardless of the approach used to calculate it; see Introduction) as a complement or an alternative to population size, depending on the study context, to assess the impact of management strategies on the population dynamics. In a small population, for example, for which a precise estimate of abundance is not a helpful indication of its status, population growth rate is a better metric for conservation. In other situations, e.g., for large harvested populations, population growth rate alone is not sufficient for making management decisions and an estimate of population size is required.

The realized $\lambda$ is easy to estimate from CR data, is robust to IDH, and we recommend its use as an indicator for management for several reasons. First, the modeling process is easy to implement because unbiased and accurate demographic estimates can be obtained with homogeneous models (ignoring IDH) that are relatively straightforward to fit. Second, estimating $\lambda$ provides some insight into the mechanisms underlying interannual abundance variations in terms of survival and recruitment, which are two vital processes that managers can control (Nichols et al. 2005). Third, it costs less time and money for managers to be aware of the presence of IDH and to use a robust population indicator, compared with trying to avoid IDH by improving monitoring protocols. Finally, in contrast to other methods based on minimal counts using groundtracking (Beyer et al. 2004) or aerial surveys (Crete and Messier 1987), our approach provides a measure of uncertainty associated with $\lambda$. Quantifying and incorporating sources of uncertainty into the decision-making process, although challenging (Regan et al. 2005), is important to balance the social and conservation risks when controlling population size.

\section{ACKNOWLEDGMENTS}

The authors are indebted to all the fieldworkers who gathered the biological samples, and to Ana Sanz Aguilar, Emmanuelle Cam, and Rachel McCrea for their comments on an earlier draft. This work was supported by a grant from the French National Game and Wildlife Agency (Office National de la Chasse et de la Faune Sauvage) and a grant from the Jeunes Chercheuses et Jeunes Chercheurs program of the French ANR (ANR-08-JCJC-0028-01).

\section{Literature Cited}

Beyer, D., T. Hogrefe, R. B. Peyton, P. Bull, J. P. Burroughs, and P. Lederle, editors. 2006. Review of social and biological science relevant to wolf management in Michigan. Michigan Department of Natural Resources, Lansing, Michigan, USA.

Beyer, D. E., B. J. Roell, and D. H. Lonsway. 2004. 2003 Survey of the gray wolf population in Michigan's Upper Peninsula. Michigan Department of Natural Resources, Marquette, Michigan, USA

Blanco, J., S. Reig, and L. De la Cuesta. 1992. Distribution, status and conservation problems of the wolf Canis lupus in Spain. Biological Conservation 60:73-80.

Blouin, M. S. 2003. DNA-based methods for pedigree reconstruction and kinship analysis in natural populations. Trends in Ecology and Evolution 18:503-511.

Boulanger, J., S. Himmer, and C. Swan. 2004. Monitoring of grizzly bear population trends and demography using DNA mark-recapture methods in the Owikeno Lake area of British Columbia. Canadian Journal of Zoology 2:1267-1277.

Breitenmoser, U. 1998. Large predators in the Alps: the fall and rise of man's competitors. Biological Conservation 83:279289.

Burnham, K. P., and D. R. Anderson. 2002. Model selection and multimodel inference: a practical information-theoretic approach. Springer-Verlag, New York, New York, USA.

Carothers, A. D. 1973. The effects of unequal catchability on Jolly-Seber estimates. Biometrics 29:79-100.

Caswell, H. 2001. Matrix population models. Second edition. Sinauer, Sunderland, Massachusetts, USA.

Chapron, G., S. Legendre, R. Ferriere, J. Clobert, and R. G. Haight. 2003. Conservation and control strategies for the wolf (Canis lupus) in western Europe based on demographic models. Comptes Rendus Biologies 326:575-587. 
Choquet, R., L. Rouan, and R. Pradel. 2009. Program ESURGE: a software application for fitting multievent models. Pages 845-865 in D. L. Thomson, E. G. Cooch, and M. J. Conroy, editors. Modeling demographic processes in marked populations. Springer, New York, New York, USA.

Ciucci, P., W. Reggioni, L. Maiorano, and L. Boitani. 2009. Long-distance dispersal of a rescued wolf from the Northern Apennines to the Western Alps. Journal of Wildlife Management 73:1300-1306.

Clark, J. D., F. T. van Manen, and M. R. Pelton. 2005. Bait stations, hard mast, and black bear population growth in Great Smoky Mountains National Park. Journal of Wildlife Management 69:1633-1640.

Coleman, K. 1998. Shyness and boldness in pumpkinseed sunfish: individual differences are context-specific. Animal Behaviour 56:927-936.

Crespin, L., R. Choquet, M. Lima, J. Merritt, and R. Pradel. 2008. Is heterogeneity of catchability in capture-recapture studies a mere sampling artifact or a biologically relevant feature of the population? Population Ecology 50:247-256.

Crespin, L., M. P. Harris, J.-D. Lebreton, and S. Wanless. 2006. Increased adult mortality and reduced breeding success with age in a population of common guillemot Uria aalge using marked birds of unknown age. Journal of Avian Biology 37:273-282.

Crete, M., and F. Messier. 1987. Evaluation of indices of gray wolf, Canis lupus, density in hardwood-conifer forests of southwestern forests of southwestern Quebec. Canadian Field-Naturalist 101:147-152.

Cubaynes, S., R. Pradel, R. Choquet, C. Duchamp, J. M. Gaillard, J.-D. Lebreton, E. Marboutin, C. Miquel, A. M. Reboulet, C. Poillot, P. Taberlet, and O. Gimenez. 2010. Importance of accounting for detection heterogeneity when estimating abundance: the case of French wolves. Conservation Biology 24:621-626.

Davis, S. A., L. K. Akison, L. N. Farroway, and G. R. Singleton. 2003. Abundance estimators and truth: Accounting for individual heterogeneity in wild house mice. Journal of Wildlife Management 67:634-645.

Devineau, O., R. Choquet, and J.-D. Lebreton. 2006. Planning capture-recapture studies: straightforward precision, bias, and power calculations. Wildlife Society Bulletin 34:10281035.

Ebert, C., D. Huckschlag, H. K. Schulz, and U. Hohmann. 2009. Can hair traps sample wild boar (Sus scrofa) randomly for the purpose of non-invasive population estimation? European Journal of Wildlife Research 56:583-590.

Ebert, C., F. Knauer, I. Storch, and U. Hohmann. 2010. Individual heterogeneity as a pitfall in population estimates based on non-invasive genetic sampling: a review and recommendations. Wildlife Biology 16:225-240.

European Commission Environment. 1992. Council Directive 92/43/EEC of 21 May 1992 on the conservation of natural habitats and of wild fauna and flora. European Commission Environment, Brussels, Belgium. 〈http://ec.europa.eu/ environment/nature/legislation/habitatsdirective/index_en. $\mathrm{htm}\rangle$

Fabbri, E., et al. 2007. From the Apennines to the Alps: colonization genetics of the naturally expanding Italian wolf (Canis lupus) population. Molecular Ecology 16:1661-1671.

Franklin, A. B., et al. 2004. Population dynamics of California Spotted Owl (Strix occidentalis occidentalis). Ornithological Monographs 54:1-110.

Harmsen, B. J., R. J. Foster, and C. Patrick Doncaster. 2010. Heterogeneous capture rates in low density populations and consequences for capture-recapture analysis of cameratrap data. Population Ecology 1-7. [doi: 10.1007/ s10144-010-0211-z]

Harrington, F. H., and L. D. Mech. 1983. Wolf pack spacing: howling as a territory-independent spacing mechanism in a territorial population. Behavioral Ecology and Sociobiology 12:161-168.

Hines, J. E., and J. D. Nichols. 2002. Investigations of potential bias in the estimation of lambda using Pradel's 1996 model for capture-recapture data. Journal of Applied Statistics 29:573-587.

Karanth, K. U., J. D. Nichols, N. S. Kumar, and J. E. Hines. 2006. Assessing tiger population dynamics using photographic capture-recapture sampling. Ecology 87:2925-2937.

Kohn, M. H., E. C. York, D. A. Kamradt, G. Haught, R. M. Sauvajot, and R. K. Wayne. 1999. Estimating population size by genotyping faeces. Proceedings of the Royal Society of London B 266:1-7.

Link, W. A., J. Yoshizaki, K. H. Pollock, and L. Bailey. 2010. Uncovering a latent multinomial: analysis of mark-recapture data with misidentification. Biometrics 66:178-185.

Lowe, W. H., M. A. McPeek, G. E. Likens, and B. J. Cosentino. 2008. Linking movement behaviour to dispersal and divergence in plethodontid salamanders. Molecular Ecology 17:4459-4469.

Lukacs, P. M., and K. P. Burnham. 2005. Review of capturerecapture methods applicable to noninvasive genetic sampling. Molecular Ecology 14:3909-3919.

Marboutin, E., and C. Duchamp. 2005. Gestion adaptative de la population de loup en France: du monitoring à l'évaluation des possibilités de prélèvements. ONCFS [Office National de la Chasse et de la Faune Sauvage] Rapport Scientifique 1:14-19.

Marucco, F., D. H. Pletscher, L. Boitani, M. K. Schwartz, K. L. Pilgrim, and J.-D. Lebreton. 2009. Wolf survival and population trend using noninvasive capture-recapture techniques in the Western Alps. Journal of Applied Ecology 46: 1003-1010.

Mech, L. D., and L. Boitani. 2003. Wolf social ecology. Pages 1-34 in L. D. Mech and L. Boitani, editors. Wolves. Behavior, ecology, and conservation. University of Chicago Press, Chicago, Illinois, USA.

Miquel, C., E. Bellemain, C. Poillot, J. Bessiere, A. Durand, and P. Taberlet. 2006. Quality indexes to assess the reliability of genotypes in studies using noninvasive sampling and multiple-tube approach. Molecular Ecology Notes 6:985988.

Mondol, S., K. U. Karanth, N. S. Kumar, A. M. Gopalaswamy, A. Andheria, and U. Ramakrishnan. 2009. Evaluation of non-invasive genetic sampling methods for estimating tiger population size. Biological Conservation 142:2350-2360.

Nichols, J. M., L. Moniz, J. D. Nichols, L. M. Pecora, and E. Cooch. 2005. Assessing spatial coupling in complex population dynamics using mutual prediction and continuity statistics. Theoretical Population Biology 67:9-21.

Patterson, B. R., and D. L. Murray. 2008. Flawed population viability analysis can result in misleading population assessment: A case study for wolves in Algonquin Park, Canada. Biological Conservation 141:669-680.

Pledger, S., K. H. Pollock, and J. L. Norris. 2003. Open capture-recapture models with heterogeneity: I. CormackJolly-Seber model. Biometrics 59:786-794.

Pradel, R. 1996. Utilization of capture-mark-recapture for the study of recruitment and population growth rate. Biometrics 52:703-709.

Pradel, R., R. Choquet, M. Lima, J. Merritt, and L. Crespin. 2009. Estimating population growth rate from capturerecapture data in presence of capture heterogeneity. Journal of Agricultural, Biological, and Environmental Statistics 15:248-258.

Rabinowitz, A., and K. A. Zeller. 2010. A range-wide model of landscape connectivity and conservation for the jaguar, Panthera onca. Biological Conservation 143:939-945.

Regan, H. M., Y. Ben-Haim, B. Langford, W. G. Wilson, P. Lundberg, S. J. Andelman, and M. A. Burgman. 2005. Robust decision-making under severe uncertainty for con- 
servation management. Ecological Applications 15:14711477.

Rutledge, L. Y., B. R. Patterson, K. J. Mills, K. M. Loveless, D. L. Murray, and B. N. White. 2009. Protection from harvesting restores the natural social structure of eastern wolf packs. Biological Conservation 143:332-339.

Skalski, J. R., A. Hoffmann, and S. G. Smith. 1993. Testing the significance of individual- and cohort-level covariates in animal survival studies. Pages 9-28 in J.-D. Lebreton and P. M. North, editors. Marked individuals in the study of bird populations. Birkhäuser Verlag, Basel, Switzerland.

Smith, D. A., K. Ralls, A. Hurt, B. Adams, M. Parker, B. Davenport, M. C. Smith, and J. E. Maldonado. 2003. Detection and accuracy rates of dogs trained to find scats of San Joaquin kit foxes (Vulpes macrotis mutica). Animal Conservation 6:339-346.

Summerlin, C. T., and J. L. Wolfe. 1973. Social influences on trap response of the cotton rat, Sigmodon hispidus. Ecology 54:1156-1159.

Taberlet, P., S. Griffin, B. Goossens, S. Questiau, V. Manceau, N. Escaravage, P. L. Waits, and J. Bouvet. 1996. Reliable genotyping of samples with very low DNA quantities using PCR. Nucleic Acids Research 24:3189-3194.

Taberlet, P., and G. Luikart. 1999. Non-invasive genetic sampling and individual identification. Biological Journal of the Linnaean Society 68:41-55.

Tavecchia, G., R. Pradel, V. Boy, A. R. Johnson, and F. Cezilly. 2001. Sex- and age-related variation in survival and cost of first reproduction in Greater Flamingos. Ecology $82: 165-174$.

Treves, A., L. Naughton-Treves, E. K. Harper, D. J. Mladenoff, R. A. Rose, T. A. Sickley, and A. P. Wydeven. 2004. Predicting human-carnivore conflict: a spatial model derived from 25 years of data on wolf predation on livestock. Conservation Biology 18:114-125.

Valière, N., L. Fumagalli, L. Gielly, C. Miquel, B. Lequette, M. L. Poulle, J. M. Weber, R. Arlettaz, and P. Taberlet. 2003. Long-distance wolf recolonization of France and Switzerland inferred from non-invasive genetic sampling over a period of 10 years. Animal Conservation 6:83-92.

Vila, C., V. Urios, and J. Castroviejo. 1994. Use of faeces for scent marking in Iberian wolves (Canis lupus). Canadian Journal of Zoology 72:374-377.

Villella, R. F., D. R. Smith, and D. P. Lemarie. 2004. Estimating survival and recruitment in a freshwater mussel population using mark-recapture techniques. American Midland Naturalist 151:114-133.

Wilson, G. J., A. C. Frantz, L. C. Pope, T. J. Roper, T. A. Burke, C. L. Cheeseman, and R. J. Delahay. 2003. Estimation of badger abundance using faecal DNA typing. Journal of Applied Ecology 40:658-666.

Wright, J. A., R. J. Barker, M. R. Schofield, A. C. Frantz, A. E. Byrom, and D. M. Gleeson. 2009. Incorporating genotype uncertainty into mark-recapture-type models for estimating abundance using DNA samples. Biometrics 65:833-840.

Zimen, E., and L. Boitani. 1975. Number and distribution of wolves in Italy. Zeitchrift für Säugetierkunde 40:102-112.

\section{APPENDIX A}

Population growth rate bias and mean-square error in the heterogeneous model and the homogeneous model fitted to simulated data (Ecological Archives A021-130-A1).

\section{APPENDIX B}

Survival and seniority bias and mean-square error in the model with heterogeneity vs. a model with homogeneity (Ecological Archives A021-130-A2). 